\title{
INNOVATION MODELLING AND WAVELET ANALYSIS OF FRACTAL PROCESSES IN BIO-IMAGING
}

\author{
Pouya Dehghani Tafti, Dimitri Van De Ville, and Michael Unser \\ Biomedical Imaging Group, EPFL, CH-1015 Lausanne, Switzerland
}

\begin{abstract}
Growth and form in biology are often associated with some level of fractality. Fractal characteristics have also been noted in a number of imaging modalities. These observations make fractal modelling relevant in the context of bio-imaging.

In this paper, we introduce a simple and yet rigorous innovation model for multi-dimensional fractional Brownian motion ( $\mathrm{fBm}$ ) and provide the computational tools for the analysis of such processes in a multi-resolution framework. The key point is that these processes can be whitened by application of the appropriate fractional Laplacian operator which has a corresponding polyharmonic wavelet. We examine the case of MRI and mammography images through comparison with theoretical results, which underline the suitability of fractal models in the study of bio-textures.
\end{abstract}

Index Terms - fractional Brownian motion, non-separable wavelets, whitening, Hurst exponent estimation.

\section{INTRODUCTION}

This paper addresses the subject of fractal analysis of two- and higher-dimensional images and datasets obtained in medical and biomedical imagery. We provide a mathematical framework wherein the problem can be rigorously formulated, and propose some computational tools for the task, which are based on multi-scale wavelet analysis.

The relevance of scale-invariance and self-similarity, central to fractal models, to the study of biomedical and other texture images has long been recognized $[1,2]$. Generally speaking, it can be argued that anatomical growth processes lead to structures that exhibit fractal statistics. This has been shown, for instance, in the case of the vasculature of the brain, where a fractal-like pattern can be identified in the tree structure of the arteries [3,4]. Additionally, the boundary between the white matter and the cerebral cortex demonstrate fractal properties (see for instance Bullmore \& al. [5]). Fractal analysis has also been applied to functional MR images [6]. Other relevant examples of medical and biomedical fractals include mammograms [7], bone structure [8], images of the cytoskeleton, and other classes of bio-textures. In addition, the $1 / \omega^{\alpha}$-like spectral decay that is directly linked to self-similarity in fractals has also been observed in fluorescence microscopy [9].

These power-law spectra that derive from self-similarity are also observed in a wide range of other applications and are typically modelled by fractional Brownian motion ( $\mathrm{fBm})$. It is not a coincidence that wavelet analysis has proved useful in the analysis of fBm (for early examples see for instance Flandrin [10] and Wornell [11]), as wavelet theory is essentially based on the concepts of multi-resolution and scaling.

This work was partially funded by the Swiss Science Foundation.
Still, an exact mathematical treatment of $\mathrm{fBm}$ and its wavelets analysis is somewhat evasive, in that useful statistically self-similar models ( $\mathrm{fBm}$ in particular) are typically non-stationary, and thereby the application of the notion of a power spectrum to them requires caution. To address this issue, we propose a distributional formulation (Section 2), in accordance with Gel'fand and Vilenkin's theory of generalized stochastic analysis [12]. This formulation, which is a multi-dimensional extension of Blu and Unser's 1-D treatment $[13,14]$, leads to an innovation model for fractional Brownian motion, which appears as fractionally integrated white noise.

Next, in order to provide computational tools for the analysis of such models, we invoke the same notions of self-similarity and scaleindependence to define a family of multi-resolution spline spaces which lead to wavelets that are intrinsically linked to the whitening operator of multi-dimensional fBm (Section 3). In contrast to the state of the art in wavelet analysis of fractal images, where separable extensions of 1-D estimators are typically used, our construction allows non-separable analysis on minimally constrained multidimensional lattices.

Finally, as a proof of potential, we employ the introduced approach, using a quincunx wavelet decomposition as per Van De Ville \& al. [15], to estimate the fractal dimensions of synthesized $\mathrm{fBm}$ samples, as well as those of T2 MRI and mammography images (Section 4).

\section{FRACTIONAL BROWNIAN MOTION}

In order to give rigorous sense to power-law spectral models, some mathematical background is essential.

\subsection{Preliminaries}

We need a means of characterizing random processes. What we propose is to use the distributional framework developed by Gel'fand and Vilenkin [12], wherein a random process $\mathfrak{X}$ is identified by the joint distribution of its "inner products" with a class of test functions $u \in \mathcal{K}$, that is, the variables

$$
\left\langle\mathfrak{X}, u_{k}\right\rangle, \quad \forall u_{k} \in \mathcal{K}, 1 \leq k \leq N, N \in \mathbb{N}_{+} .
$$

Such random variables may be regarded as measurements or observations of the random process $\mathfrak{X}$. They are comparable to samples $\mathfrak{X}\left(\boldsymbol{x}_{k}\right), \boldsymbol{x}_{k} \in \mathbb{R}^{d}$, of the random process considered in the classical theory.

Given an operator $\mathrm{A}$ on $\mathcal{K}$ and its adjoint $\mathrm{A}^{*}$, the application of A on $\mathfrak{X}$ is defined by

$$
\langle\mathrm{A} \mathfrak{X}, u\rangle \stackrel{\text { def }}{=}\left\langle\mathfrak{X}, \mathrm{A}^{*} u\right\rangle .
$$

Also, the Fourier transform $\hat{\mathfrak{X}}$ of $\mathfrak{X}$ is defined by the Parseval identity:

$$
\langle\hat{\mathfrak{X}}, \hat{u}\rangle \stackrel{\text { def }}{=}(2 \pi)^{d}\langle\mathfrak{X}, u\rangle
$$




\subsection{Characterization of $\mathrm{fBm}$ processes}

A fractional Brownian motion $\mathfrak{B}_{H}$ with Hurst parameter $H$ is a zeromean nonstationary process that is classically defined by its variogram, which describes the variance of its increments:

$$
\mathbf{E}\left\{\left|\mathfrak{B}_{H}\left(\boldsymbol{x}_{a}\right)-\mathfrak{B}_{H}\left(\boldsymbol{x}_{b}\right)\right|^{2}\right\}=\sigma^{2}\left\|\boldsymbol{x}_{a}-\boldsymbol{x}_{b}\right\|_{2}^{2 H} .
$$

( $\sigma$ is an arbitrary constant.)

To give a distributional definition of these processes, we first introduce the operator $\Delta^{-\gamma}$ and its adjoint $\Delta^{-\gamma}$, which, respectively, are particular left and right inverses of the (normalized) fractional Laplacian

$$
\Delta^{\gamma} \stackrel{\mathscr{F}}{\sim}\|\boldsymbol{\omega}\|^{2 \gamma} .
$$

The left and right inverses are defined in the Fourier domain as follows:

$$
\begin{aligned}
& \grave{\Delta}^{-\gamma} f(\boldsymbol{x}) \stackrel{\text { def }}{=} \\
& (2 \pi)^{-d} \int_{\mathbb{R}^{d}} \mathrm{~d} \boldsymbol{\omega} \mathrm{e}^{\mathrm{j} \boldsymbol{x}^{\top} \boldsymbol{\omega}} \frac{\hat{f}(\boldsymbol{\omega})-\sum_{|\boldsymbol{k}| \leq\left\lfloor 2 \gamma-\frac{d}{2}\right\rfloor} \hat{f}^{(\boldsymbol{k})}(\mathbf{0}) \frac{\boldsymbol{\omega}^{\boldsymbol{k}}}{\boldsymbol{k} !}}{\|\boldsymbol{\omega}\|^{2 \gamma}} ; \\
& \dot{\Delta}^{-\gamma} f(\boldsymbol{x}) \stackrel{\text { def }}{=} \\
& (2 \pi)^{-d} \int_{\mathbb{R}^{d}} \mathrm{~d} \boldsymbol{\omega} \frac{\mathrm{e}^{\mathrm{j} \boldsymbol{x}^{\top} \boldsymbol{\omega}}-\sum_{|\boldsymbol{k}| \leq\left\lfloor 2 \gamma-\frac{d}{2}\right\rfloor} \frac{\mathrm{j}^{|\boldsymbol{k}|} \boldsymbol{x}^{\boldsymbol{k}} \boldsymbol{\omega}^{\boldsymbol{k}}}{\boldsymbol{k} !}}{\|\boldsymbol{\omega}\|^{2 \gamma}} \hat{f}(\boldsymbol{\omega}) .
\end{aligned}
$$

It can then be shown that the classical definition of a fractional Brownian motion $\mathfrak{B}_{H}$ is equivalent to the following definition

$$
\mathfrak{B}_{H} \stackrel{\text { def }}{=} \epsilon_{H}^{\prime} \dot{\Delta}^{-\frac{H}{2}-\frac{d}{4}} \mathfrak{W}
$$

which simply states that $\mathrm{fBm}$ is obtained by suitable fractional integration of a white Gaussian noise process $\mathfrak{W}$ with variance $\sigma^{2}$. Here $\epsilon_{H}^{\prime}$ is an $H$-dependent factor given by

$$
\epsilon_{H}^{\prime 2}=-2^{2 H+d-1} \pi^{d / 2} \frac{\Gamma\left(H+\frac{d}{2}\right)}{\Gamma(-H)} .
$$

(The proof is technical and will be published elsewhere [16].) As an important and immediate consequence we can see that the fractional Laplacian operator $\Delta^{\frac{H}{2}+\frac{d}{4}}$ whitens an $\mathrm{fBm}$ field of exponent $H$, that is,

$$
\Delta^{\frac{H}{2}+\frac{d}{4}} \mathfrak{B}_{H}=\epsilon_{H}^{\prime} \mathfrak{W} .
$$

The appearance of the Laplacian and its inverses in the definition of $\mathrm{fBm}$ is not arbitrary, as the fractional Laplacian is practically the only real convolution operator that exhibits invariance to rotation and scaling $[17,18]$.

\section{POLYHARMONIC MULTI-RESOLUTION ANALYSIS}

The same operators that characterize our stochastic processes of interest can also be used to define families of polyharmonic splines and their corresponding multi-resolution spaces spanned by B-spline functions distributed over a $d$-dimensional lattice $\mathrm{Q} \mathbb{Z}^{d} \stackrel{\text { def }}{=}\{\mathrm{Q} \boldsymbol{k} \mid \boldsymbol{k} \in$ $\left.\mathbb{Z}^{d}\right\}$. ( $Q$ is the lattice generator matrix.) This is done by defining polyharmonic splines as solutions to a fractional Poisson equation of the following construction:

$$
\Delta^{\gamma} s(\boldsymbol{x})=\sum_{\boldsymbol{k} \in \mathbb{Z}^{d}} c_{\boldsymbol{k}} \delta(\boldsymbol{x}-\mathrm{Q} \boldsymbol{k})
$$

$(\delta(\cdot)$ denotes Dirac's delta.) Polyharmonic B-splines are particular solutions of the above equation, with coefficients $c_{k}$ that define a discrete localization filter whose frequency response (denoted $\hat{V}_{Q}^{\lambda}$ ) approximates $\hat{\Delta}^{\gamma}(\boldsymbol{\omega})=\|\boldsymbol{\omega}\|^{2 \gamma}$ around $\boldsymbol{0}$. Such a filter can be constructed by choosing a frequency response of the form

$$
\hat{\mathrm{V}}_{\mathrm{Q}}^{\gamma}(\boldsymbol{\omega})=\left[\frac{4}{\mu^{2}} \sum_{1 \leq i<N} \sin ^{2}\left(\frac{\boldsymbol{y}_{i}^{\top} \mathrm{Q}^{\top} \boldsymbol{\omega}}{2}\right)\right]^{\gamma},
$$

where the multi-integer vectors $\boldsymbol{y}_{1}, \ldots, \boldsymbol{y}_{N} \in \mathbb{Z}^{d}$ are such that the (compulsorily linearly independent) vectors $\mathrm{Q} \boldsymbol{y}_{1}, \ldots, \mathrm{Q} \boldsymbol{y}_{N}$ generate the lattice $\mathrm{Q} \mathbb{Z}^{d}$ and form a tight-frame for $\mathbb{R}^{d}$ with frame constant $\mu^{2}$. A convolution of such a filter with an invertible stable filter also provides an acceptable localization.

The above formulation results in the following Fourier domain definition of the polyharmonic B-spline function $\phi_{2 \gamma}(\boldsymbol{x})$ :

$$
\hat{\phi}_{2 \gamma}(\boldsymbol{\omega})=\frac{\hat{\mathrm{V}}_{Q}^{\gamma}(\boldsymbol{\omega})}{\|\boldsymbol{\omega}\|^{2 \gamma}}
$$

which generalizes Rabut's $\gamma$-harmonic B-splines $[15,19] . \quad$ It is possible to show that for $\gamma>\frac{d}{4}$ the lattice shifts of $\phi_{2 \gamma}$ are squareintegrable and satisfy the necessary conditions for constituting a Mallat-type multi-resolution analysis (MRA) [20-22], meaning that they

a) form a partition of unity;

b) fulfil a two-scale relation of the form

$$
\phi_{2 \gamma}\left(\mathrm{QD}^{-1} \mathrm{Q}^{-1} \boldsymbol{x}\right)=\sum_{\boldsymbol{k} \in \mathbb{Z}^{d}} h_{\boldsymbol{k}} \phi_{2 \gamma}(\boldsymbol{x}-\mathrm{Q} \boldsymbol{k})
$$

with $h \in \ell^{1}\left(\mathbb{Z}^{d}\right)$, for any integer subsampling matrix D such that $\mathrm{QDQ}^{-1}$ corresponds to a similarity transform; and

c) generate a Riesz basis for their $\ell^{2}$ span.

The multi-resolution spaces are defined by

$$
\mathcal{V}_{n} \stackrel{\text { def }}{=}\left\{\sum_{k \in \mathbb{Z}^{d}} c_{\boldsymbol{k}} \phi_{2 \gamma}\left(\mathrm{QD}^{-n} \mathrm{Q}^{-1} \cdot-\mathrm{Q} k\right) \mid c \in \ell^{2}\left(\mathbb{Z}^{d}\right)\right\} .
$$

These spaces are nested, i.e.,

$$
\{0\} \subset \cdots \subset \mathcal{V}_{1} \subset \mathcal{V}_{0} \subset \mathcal{V}_{-1} \subset \cdots \subset \mathrm{L}^{2}\left(\mathbb{R}^{d}\right)
$$

Polyharmonic wavelet bases are lattice-shift-invariant sets spanning the orthogonal complements in the series of nested multiresolution spaces. The significant property of these wavelets is that they approximate the fractional Laplacian $\Delta^{\gamma}$ at low frequencies, in the sense that for any semi-orthogonal polyharmonic wavelet $\psi_{2 \gamma}$ we have

$$
\psi_{2 \gamma}(\boldsymbol{x})=\Delta^{\gamma} \eta(\boldsymbol{x})
$$

with $\eta(\boldsymbol{x})$ (the smoothing kernel) having a Sobolev exponent of no less than $4 \gamma-\frac{d}{2}$.

\section{WAVELET ANALYSIS OF FBM}

Owing to the Laplacian-like behaviour of the wavelets at low frequencies (Eqn (3)), we can show the following results about the wavelet transform coefficients of an $\mathrm{fBm}$ process, given by

$$
w_{n}[\boldsymbol{k}]=\left\langle\mathfrak{B}_{H},|\mathrm{D}|^{-\frac{n}{2}} \psi_{2 \gamma}\left(\mathrm{QD}^{-n} \mathrm{Q}^{-1} \boldsymbol{x}-\mathrm{Q} \boldsymbol{k}\right)\right\rangle .
$$


a) The polyharmonic spline wavelet transform of order $2 \gamma \geq H+\frac{d}{2}$ maps the non-stationary process $\mathfrak{B}_{H}$ into a series of stationary (discrete) Gaussian processes, which correspond to sequences of samples of a stationary process with power spectrum $\epsilon_{H}^{\prime}\|\omega\|^{2 \gamma-H-\frac{d}{2}}|\hat{\eta}(\boldsymbol{\omega})|^{2}$.

b) The variance of the polyharmonic wavelet coefficients of $\mathfrak{B}_{H}$ depends exponentially on the Hurst exponent and the scale $n$ :

$$
\mathbf{E}\left\{w_{n}^{2}[\boldsymbol{k}]\right\}=|\mathrm{D}|^{\frac{(2 H+d) n}{d}} \mathbf{E}\left\{w_{0}^{2}[\boldsymbol{k}]\right\} .
$$

The properties just mentioned allow us to generalize wavelet estimators of the Hurst parameter that exist in the literature [10,20,23] to the multi-dimensional case. We may take the logarithm of the sides of Eqn (4), thus obtaining the relation

$$
\log _{\sqrt[d]{|\mathrm{D}|}}\left(\mathbf{E}\left\{w_{n}^{2}[\boldsymbol{k}]\right\}\right)=(2 H+d) n+C
$$

whereby an estimate of the Hurst parameter may by found via linear regression of sub-band variances in a logarithmic scale. ( $C$ in the above equation is a computable constant that depends on the particular wavelet used in the analysis.)

\section{SIMULATION AND EXPERIMENTS}

To verify the estimation procedure described above, instances of two-dimensional $\mathrm{fBm}$ were created by non-stationary Fourier domain filtering of white Gaussian noise as per $\$ 2.2$ and analysed using the quincunx isotropic polyharmonic wavelets of Van De Ville \& al. [15] (Figs 1(a) and 1(d)). A fast FFT-based implementation of the wavelet transform of sufficient order was used. The quincunx subsampling scheme utilized provides a more gradual scale progression in comparison with dyadic subsampling, thereby affording more data points for the regression step. (A second advantage is that the quincunx design involves only a single mother-wavelet.) Results for $100 \mathrm{fBm}$ images of dimensions $512 \times 512$ and three different values of $H$ are summarized in Table 1, demonstrating the robust (albeit biased) nature of the estimator.

Table 1. Wavelet-based estimation of $H$ (100 realizations)

\begin{tabular}{|c|c|c|}
\hline \hline True value & Mean $H_{\text {est }}$ & Standard dev. $H_{\text {est }}$ \\
\hline 0.3 & 0.285 & 0.012 \\
0.6 & 0.584 & 0.012 \\
0.9 & 0.884 & 0.013 \\
\hline \hline
\end{tabular}

A similar analysis was applied to T2 MRI (with boundaries and background removed) and mammograms (Figs 1(b) and 1(c)). In both cases, strong agreement with the fractal model (indicated by the linear growth of the wavelet coefficient variances in the logarithmic scale) was observed (Figs 1(e) and 1(f)). As already noted in the introduction, some justification for this behaviour can be found in the relation between growth processes and fractals (particularly in the case of the branching of the arteries in the brain, as T2 MRI can provide an indirect measurement of the concentration of oxygenated blood). In addition, these observations can be taken as further evidence for the relevance of fractal models and fractal analysis in medical and biomedical applications.

\section{CONCLUSION}

Motivated by the ubiquity of bio-fractals and the perceived importance of fractal models in medical and biomedical imaging, we proposed a simple innovation model of $\mathrm{fBm}$ in concordance with Gel'fand and Vilenkin's theory of generalized stochastic processes [12], and suggested a family of computational tools - rooted in multi-resolution and multi-scale analysis - for the fractal analysis of multi-dimensional data. The pertinence of the discussed models, and the suitability of the proposed computational tools was verified by their application to MRI and mammography images, which displayed clear agreement in behaviour with theoretical predictions. The tools and framework reviewed in this paper show a great potential for further investigation in biomedical imaging applications, and in particular, in the analysis of bio-textures obtained through different multi-dimensional bio-imaging modalities.

\section{REFERENCES}

[1] T. Lundahl, W. J. Ohley, S. M. Kay, and R. Siffert, "Fractional brownian motion: A maximum likelihood estimator and its application to image texture," IEEE Trans. Med. Imag., vol. 5, pp. 152-161, 1986.

[2] B. Pesquet-Popescu and J. Lévy Véhel, "Stochastic fractal models for image processing," IEEE Signal Processing Mag., vol. 19 , no. 5, pp. 48-62, 2002.

[3] L. Risser, F. Plouraboue, A. Steyer, P. Cloetens, G. Le Duc, and C. Fonta, "From homogeneous to fractal normal and tumorous microvascular networks in the brain," J. Cereb. Blood Flow Metab., vol. 27, pp. 293-303, 2007.

[4] S. Heinzer, T. Krucker, M. Stampanoni, R. Abela, E. P. Meyer, A. Schuler, P. Schneider, and R. Muller, "Hierarchical microimaging for multiscale analysis of large vascular networks," NeuroImage, vol. 32, no. 2, pp. 626-636, 2006.

[5] E. Bullmore, M. Brammer, I. Harvey, R. Persaud, R. Murray, and M. Ron, "Fractal analysis of the boundary between white matter and cerebral cortex in magnetic resonance images: a controlled study of schizophrenic and manic-depressive patients," Psychol. Med., vol. 24, no. 3, pp. 771-781, 1994.

[6] V. Maxim, L. Sendur, J. Fadili, J. Suckling, R. Gould, R. Howard, and E. Bullmore, "Fractional Gaussian noise, functional MRI and Alzheimer's disease," NeuroImage, vol. 25, no. 1, pp. 141-158, 2005.

[7] H. Li, K.J.R. Liu, and S.C.B. Lo, "Fractal modelling and segmentation for the enhancement of microcalcifications in digital mammograms," IEEE Trans. Med. Imag., vol. 16, no. 6, pp. 785-798, 1997.

[8] L. Pothuaud, E. Lespessailles, R. Harba, R. Jennane, V. Royant, E. Eynard, and C. L. Benhamou, "Fractal analysis of trabecular bone texture on radiographs: Discriminant value in postmenopausal osteoporosis," Osteoporos. Int., vol. 8, pp. 618-625, 1998.

[9] D. Sage, F.R. Neumann, F. Hediger, S.M. Gasser, and M. Unser, "Automatic tracking of individual fluorescence particles: Application to the study of chromosome dynamics," IEEE Trans. Image Processing, vol. 14, pp. 1372-1383, 2005.

[10] P. Flandrin, "Wavelet analysis and synthesis of fractional Brownian motion," IEEE Trans. Inform. Theory, vol. 38, no. 2, pp. 910-916, 1992. 


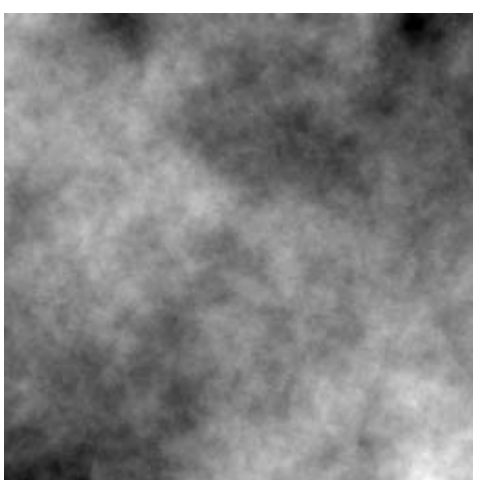

(a) synthesized fBm $(H=0.60)$

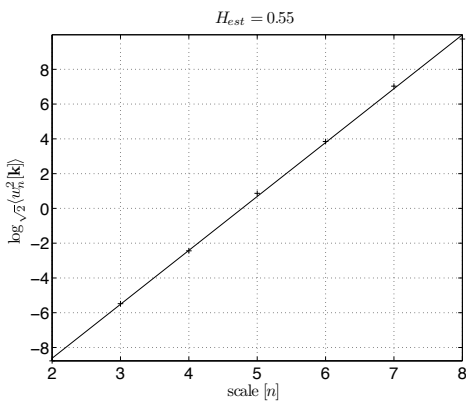

(d) $H_{\text {est }}=0.55$

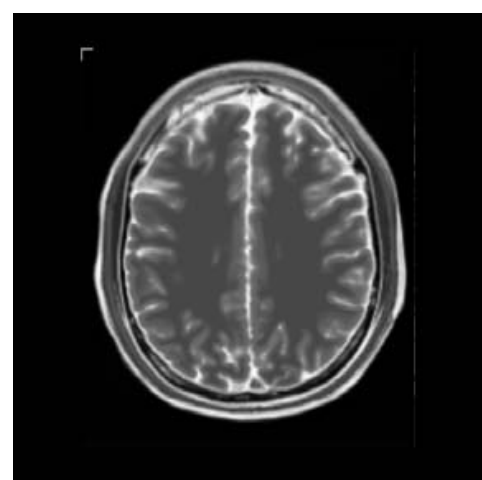

(b) T2 MRI

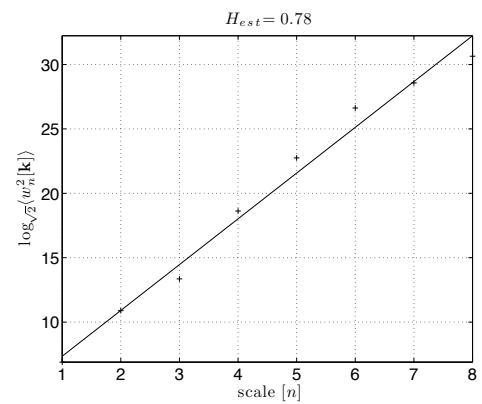

(e) $H_{\text {est }}=0.78$

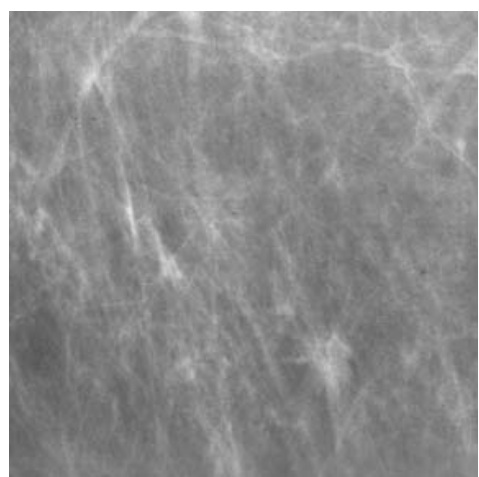

(c) mammogram

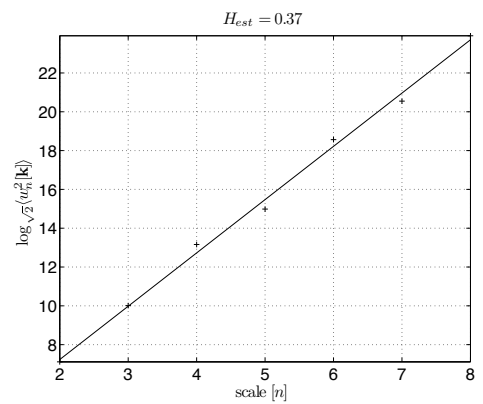

(f) $H_{\text {est }}=0.37$

Fig. 1. (a), (b), and (c): input images. (d), (e), and (f): regression plots and $H$ estimates according to (5). (T2 MRI image obtained from the BrainWeb project [24]. Mammogram courtesy of the Digital Database for Screening Mammography (DDSM) [25].)

[11] G. W. Wornell, "Wavelet-based representations for the $1 / f$ family of fractal processes," Proc. IEEE, vol. 81, no. 10, pp. 1428-1450, 1993.

[12] I. M. Gel'fand and N.Ya. Vilenkin, Applications of Harmonic Analysis, vol. IV of Generalized Functions, Academic Press, 1964, translated by Amiel Feinstein.

[13] T. Blu and M. Unser, "Self-similarity: Part II-Optimal estimation of fractal processes," IEEE Trans. Signal Processing, vol. 55, no. 4, 2007.

[14] M. Unser and T. Blu, "Self-similarity: Part I-Splines and operators," IEEE Trans. Signal Processing, vol. 55, no. 4, pp. 1352-1363, 2007.

[15] D. Van De Ville, T. Blu, and M. Unser, "Isotropic polyharmonic B-Splines: Scaling functions and wavelets," IEEE Trans. Image Process., vol. 14, no. 11, pp. 1798-1813, November 2005 .

[16] P.D̃. Tafti, D. Van De Ville, and M. Unser, "Invariances, laplacian-like wavelet bases, and the whitening of fractal processes," submitted.

[17] J. Duchon, "Splines minimizing rotation-invariant semi-norms in Sobolev spaces," in Constructive Theory of Functions of Several Variables, A. Dold and B. Eckmann, Eds., vol. 571 of Lecture Notes in Mathematics, pp. 85-100. Springer-Verlag, 1977.

[18] J. Kybic, T. Blu, and M. Unser, "Generalized sampling: A variational approach-Part I: Theory," IEEE Trans. Signal Processing, vol. 50, no. 8, pp. 1965-1976, August 2002.
[19] C. Rabut, "Elementary m-harmonic cardinal B-splines," $\mathrm{Nu}$ mer. Algorithms, vol. 2, pp. 39-61, 1992.

[20] S. Mallat, "A theory for multiresolution signal decomposition: The wavelet representation," IEEE Trans. Pattern Anal. Machine Intell., vol. 11, no. 7, July 1989.

[21] M. Unser and T. Blu, "Wavelet theory demystified," IEEE Trans. Signal Processing, vol. 51, no. 2, pp. 470-483, February 2003.

[22] C. Micchelli, C. Rabut, and F. I. Utreras, "Using the refinement equation for the construction of pre-wavelets III: Elliptic splines," Numer. Algorithms, vol. 1, no. 3, pp. 331-352, 1991.

[23] D. Veitch and P. Abry, "A wavelet-based joint estimator of the parameters of long-range dependence," IEEE Trans. Inform. Theory, vol. 45, no. 3, pp. 878-897, 1999.

[24] R.K.-S. Kwan, A.C. Evans, and G.B. Pike, "MRI simulationbased evaluation of image-processing and classification methods," IEEE Trans. Med. Imag., vol. 18, no. 11, pp. 1085-1897, 1999.

[25] M. Heath, K. Bowyer, D. Kopans, R. Moore, and W. P. Kegelmeyer, "The digital database for screening mammography," in Proc. Fifth International Workshop on Digital Mammography, M. J. Yaffe, Ed., 2001, pp. 212-218. 